\title{
Fondements et principes de la reproduction humaine, 1993-1998
}

Étienne-Émile Baulieu

\section{(2) OpenEdition \\ 1 Journals}

Édition électronique

URL : https://journals.openedition.org/annuaire-cdf/1063

DOI : 10.4000/annuaire-cdf. 1063

ISBN : 978-2-7226-0325-7

ISSN : 2109-9227

Éditeur

Collège de France

Édition imprimée

Date de publication : 1 avril 2013

Pagination : 763-766

ISBN : 978-2-7226-0198-7

ISSN : 0069-5580

\section{Référence électronique}

Étienne-Émile Baulieu, « Fondements et principes de la reproduction humaine, 1993-1998 », L'annuaire du Collège de France [En ligne], 112 | 2013, mis en ligne le 22 novembre 2013, consulté le 22 août 2022. URL : http://journals.openedition.org/annuaire-cdf/1063; DOI : https://doi.org/10.4000/annuaire-cdf. 1063 


\title{
PROFESSEURS HONORAIRES
}

\author{
ACTIVITÉS, PUBLICATIONS \\ M. Étienne-Émile BAULIEU, membre de l'Institut \\ (Académie des sciences)
}

Fondements et principes de la reproduction humaine, 1993-1998

\section{ACTIVITÉS}

(Ce résumé inclut les travaux accomplis entre les mois de juillet 2011 et juin 2012.)

En continuité des recherches menées au cours de l'année précédente, nous avons principalement développé les études neurophysiopathologiques dérivées de nos observations initiales sur les «neurostéroïdes»: Recent Progress in Hormone Research, 1997 [1].

Elles consistent d'une part à rechercher une nouvelle approche prédictive et thérapeutique de la maladie d'Alzheimer et des autres démences tauopathiques, et d'autre part à développer nos études permettant d'aborder la très difficile question du traitement des états dépressifs. Les deux domaines sont étudiés par deux groupes de collaborateurs, l'un dépendant de l'INSERM et soutenu par une fondation récente, l' "Institut Baulieu », et l'autre développant ses activités dans le cadre de la petite société biotechnologique «MAPREG » que je préside.

\section{INSERM et les fondations; maladie d'Alzheimer et démences tauopathiques}

Nous continuons sans interruption à étudier les anomalies de la protéine Tau en tant que cible principale de nos efforts thérapeutiques. Les éléments principaux 
publiés antérieurement par Chambraud et collaborateurs, 2010 [2] ont été confirmés par des études approfondies utilisant des dérivés de la protéine Tau en interaction avec la protéine FKBP52 dont nous avions préalablement souligné l'importance potentielle, ce qui n'a été démenti dans aucune de nos études. D'ailleurs, nous avons pu publier les résultats d'observations faites sur les cerveaux de personnes décédées de maladie d'Alzheimer et de la principale autre tauopathie FTDP-17 (Giustiniani et collaborateurs 2012 [3]) qui correspondent remarquablement à nos observations in vitro. Les travaux actuels se poursuivent tout particulièrement en tenant compte des observations récentes indiquant le fonctionnement «prion-like » de la protéine Tau et le rôle qu'y pourrait jouer FKBP52. Ils nécessitent la synthèse de Tau et FKBP52 strictement pures, et l'utilisation de plusieurs types de protéinekinases et d'enzymes protéolytiques. Quand on note que FKBP52 est une protéine «drugable », c'est-à-dire susceptible de lier des ligands dérivés du FK506 mais sans activité immunosuppressive, on conçoit l'importance de nos études avec des molécules empruntées à des sociétés pharmaceutiques qui les avaient synthétisées pour d'autres recherches dans des domaines différents (Astellas, Pfizer, Biotica). La sélection des ligands se fait initialement par les résultats des travaux de résonance magnétique nucléaire (RMN) dans le laboratoire des Drs Guy Lippens et Isabelle Landrieu, département de biochimie de l'université de Lille, avec qui nous collaborons. L'étude biologique des effets sur Tau de l'interaction de FKBP52 avec des ligands pharmacologiques se fait sur le poisson-zèbre, dont nous avons initié l'utilisation dans ce domaine avec le Dr. Marcel Tawk (publication en préparation [4]). Avec cette approche permettant d'obtenir un modèle quelques jours après l'introduction d'un candidat médicament au niveau de l'œuf, nous avons pu confirmer in vivo le concept d'interaction FKBP52-Tau. De plus, en introduisant une protéine Tau anormale humaine, nous avons déjà obtenu la preuve d'un effet possible de FKBP52 sur une «tauopathie animale ». D'autre part, également pour tester les molécules potentiellement de ligands de FKBP52, nous avons développé et étudié, en collaboration avec la Pr. Marie-Christine Pardon, qui travaille à Nottingham dans le département de psychiatrie de l'université, des modèles de souris tauopathiques (en particulier hTau où la protéine de souris est remplacée par son équivalent humain). Les premiers résultats indiquent l'évolution de nos deux protéines en interaction selon l'âge. De plus, nous développons une souris FKBP52-KO que nous comptons croiser avec les souris hTau. Les études avec le poisson-zèbre sont, grâce à la rapidité du développement de l'animal, celles qui nous indiqueront les ligands potentiellement intéressants à étudier avec les modèles murins.

Nous avons aussi au programme de ce thème une recherche destinée à obtenir une symptomatologie prédictive de risque d'Alzheimer ou autres tauopathies. Sur la base des observations déjà obtenues dans le liquide céphalo-rachidien (LCR) des patients (Tau élevée et $A \beta$ abaissée [3]), nous pensons développer les résultats des mesures cérébrales de FKBP52 pour mettre au point une évaluation de cette protéine dans le LCR de sujets à différents stades de la maladie. En principe, un abaissement du taux normal de FKBP52 dans le LCR pourrait être annonciateur de pathologie. Une collection de 700 échantillons obtenus du Pr. Bruno Vellas chez des patients soigneusement étudiés cliniquement, doit nous permettre d'obtenir un résultat en deux ou trois ans sur le risque de maladie (ce travail est dirigé par les Drs Marc Roger et Najiba Lahlou, qui travaillent dans le cadre du laboratoire de biochimie hormonale à l'hôpital Cochin). 
Ces recherches ont été possibles grâce à l'appui de la fondation Vivre longtemps, de l'Institut Baulieu et, pour leur gestion, de la Fondation des Caisses d'épargne pour la solidarité et de la Fondation nationale de gérontologie. Nous avons reçu des subventions de l'Institut Mérieux et de la Fondation Claude Pompidou. Plusieurs généreux donateurs nous ont aussi aidés directement.

\section{MAPREG. Une «biotech » ciblant les microtubules cérébraux avec des dérivés stéroïdes. Traumatismes cérébraux et syndromes dépressifs}

Certains neurostéroïdes [1] agissent au niveau de la protéine MAP2, associée aux microtubules et stimulée par la prégnènolone et certains de ses dérivés. Nos recherches portent sur deux catégories de troubles pathologiques, ceux qui résultent des traumatismes du système nerveux central et ceux qui sont associés aux états dépressifs.

L'essentiel des recherches, dans les deux cas, sont menées au sein de MAPREG avec le dérivé MAP4343 dont l'activité est très semblable à celle de la prégnènolone elle-même et dont la structure (3-méthoxy-prégnènolone) exclut la transformation en composé(s) hormonalement actif(s) difficile(s) à utiliser cliniquement.

1. La société SANOFI, intéressée par nos résultats sur les traumatismes de la moelle épinière, a longuement analysé notre projet de phase II mise au point avec le Pr. Hugues Pascal-Moussellard, chef de service de traumatologie de la Pitié-Salpêtrière, et spécialisé dans les lésions de la colonne vertébrale. Le développement clinique mis au point avec les neurologues de la Salpêtrière (responsable Dr Jean-Christophe Corvol) a été présenté au cours d'une «due diligence ». Les résultats sont toujours en discussion quant à leur développement chez SANOFI, et des projets d'amplification des études concernant les traumatismes du cerveau sont en cours.

2. Les anomalies de la tubuline accompagnant les états dépressifs expérimentaux mis en évidence par le Dr Massimiliano Bianchi dans ses travaux en Grande-Bretagne incluent les effets du MAP4343, depuis l'engagement de ce chercheur comme responsable du domaine chez MAPREG. Les résultats d'un programme de deux ans et demi «DEPSTER », accordé par EUROSTARS, sont favorables. Ils concernent des recherches expérimentales en comparaison avec les composés anti-dépresseurs actuellement utilisés. MAP4343, sur le plan des comportements comme biochimiquement sur les altérations de la tubuline, s'est avéré très actif chez le rat et le toupaye de Belanger. Plusieurs sociétés pharmaceutiques s'intéressent à nos résultats depuis notre première publication (Bianchi et Baulieu, 2012 [5]), alors que la plupart des compagnies pharmaceutiques ont décidé d'abandonner le domaine de la dépression compte tenu de l'absence de nouvelles options mécanistiques pour les traitements.

3. Nouveaux dérivés. Les recherches avec un nouveau dérivé de MAP4343 ont abouti à la rédaction préliminaire de sa structure nouvelle (pour une demande de brevet). Le développement de ce composé doit permettre une alternative à MAP4343 dans certaines des indications citées plus haut.

\section{ACTIVITÉS DIVERSES}

Président du conseil scientifique d' «Équilibres et populations ». 
Président de la Fondation des Amis des sciences (Académie des sciences).

Président du sous-groupe médical « Solidarité Japon », institué par l'Académie des sciences à la suite de la catastrophe provoquée par le tremblement de terre et le tsunami de Fukushima. Le rapport est paru aux Éditions EDP Sciences [6].

\section{Références}

[1] Baulieu E.E., « Neurosteroids : of the nervous system, by the nervous system, for the nervous system », in Conn P.M. (éd.), Recent Progress in Hormone Research, The Endocrine Society Press, Bethesda, 52, 1997, 1-32.

[2] Chambraud B., Sardin E., Giustiniani J., Dounane O., Schumacher M., Goedert M. et Baulieu E.E., "A role for FKBP52 in Tau protein function », Proc. Natl. Acad. Sci. USA, $107,2010,2658-2663$.

[3] Giustiniani J., Sineus M., Sardin E., Dounane O., Panchal M., Sazdovitch V., Duyckaerts C., Chambraud B. et Baulieu E.E., «Decrease of the immunophilin FKBP52 accumulation in human brains of Alzheimer's disease and FTDP-17 », Journal of Alzheimer Disease, 29, 2012, 471-483.

[4] Chambraud B., Sardin E., Nakatani H., Paquet D., Baulieu E.E. et Tawk M., «FKBP52 plays an essential role in driving axonal outgrowth and controls motility and spinal motor axonal morphology via inhibition of pathological Tau activity in zebrafish », publication en préparation.

[5] Bianchi M. et Baulieu E.E., «3 3 -methoxy-pregnenolone (MAP4343) as an innovative therapeutic approach for depressive disorders », Proc. Natl. Acad. Sci. USA, 109, 2012, $1713-$ 1718.

[6] Groupe de travail Solidarité Japon présidé par Alain Carpentier, avec Étienne-Émile Baulieu, L'accident majeur de Fukushima. Considérations sismiques, nucléaires et médicales. Académie des sciences, EDP Sciences, 2012.

\section{Yves BONNEFOY}

\section{Études comparées de la fonction poétique, 1981-1993}

\section{PRINCIPALES PUBlications ET ACTIVITÉS DEPUIS LE $1^{\text {er }}$ OCTOBRE 2011}

\section{Livres}

Bonnefoy Y., Sous le signe de Baudelaire, Gallimard, 2011, 414 p.

Bonnefoy Y., Je vois sans yeux et sans bouche je crie, 24 sonnets de Pétrarque, avec des dessins de Gérard Titus-Carmel, Galilée, 2011, 80 p.

Bonnefoy Y., Peintures murales de la France gothique, nouvelle édition, ELLUG, Université Stendhal, Grenoble, 2012, 159 p., avec 66 photographies de Pierre Devinoy.

Bonnefoy Y., L'inachevable, Entretiens sur la poésie, 1990-2010, nouvelle édition, Le livre de poche, avril 2012, $528 \mathrm{p}$.

Bonnefoy Y., Plusieurs raisons de peindre des arbres, Éditions de Corlevour, 2012, 80 p, avec des peintures et dessins d'Agnès Prévost. 